\title{
SAPHO SYNDROME: A THERAPEUTIC CHALLENGE
}

Déborah Lobato Guimarães ${ }^{1, \star}$, Lucas Leonardo de Castro Borges ${ }^{1}$, Hyggor Henrique Bego ${ }^{2}$

1.Hospital Felício Rocho Hospital, Belo Horizonte (MG), Brazil. 2.Universidade José do Rosário Vellano, Belo Horizonte (MG), Brazil.

*Corresponding author: deborahfcmmg@gmail.com

\section{BACKGROUND}

Synovitis, acne, pustulosis, hyperostosis, and osteitis (SAPHO) syndrome is a rare, inflammatory disorder of bone, joints and skin. Individuals between the ages of 30 and 50 years seem most frequently affected, but children and young adults have also been reported. There is an apparent female predominance, particularly among patients less than 30 years of age at onset. In this report of two cases, we will present the complexity of the therapeutic approach of these patients.

\section{CASE REPORT}

Case 1

A 32-year-old female presented with an inflammatory low back pain with 7 years of evolution, complicated by diffuse pain, especially rib cage and left sternoclavicular joint (Fig. 1), in addition to erythematous scaling plantar exanthem and acneiform lesions. Since sacroiliitis was suggested by magnetic resonance imaging (MRI) (Fig. 2), it was important to differentiate the condition from axial spondyloarthritis. Patient did not respond to nonsteroidal anti-inflammatory drugs (NSAIDs), and several immunosuppressants, namely, methotrexate, sulfasalazine, azathioprine, etanercept and adalimumab. A partial response to pamidronate treatment was observed, although the patient requires adjuvant pain therapy and is followed up in a specialized pain clinic.

Case 2

A 15-year-old male presented with severe acneiform lesions experienced treatment without satisfactory response to tetracycline and isotretinoin, progressed with severe pain in the right hip and knee, in addition of the third and fourth left sternocostal joint (Fig. 3). He evolved with good response to prednisone $20 \mathrm{mg}$ and NSAIDs. Due to peripheral involvement, methotrexate was associated and the prednisone was tapered.

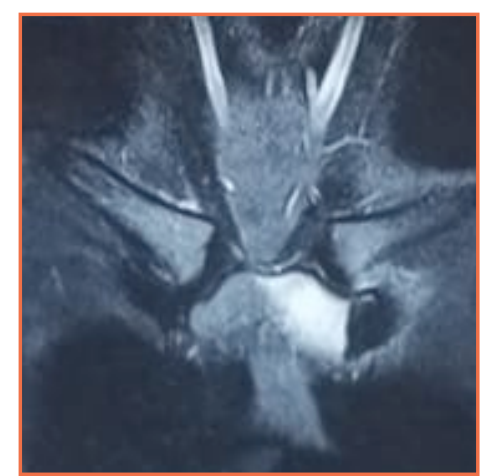

Figure 1. Sternum bone marrow edema (BMD).

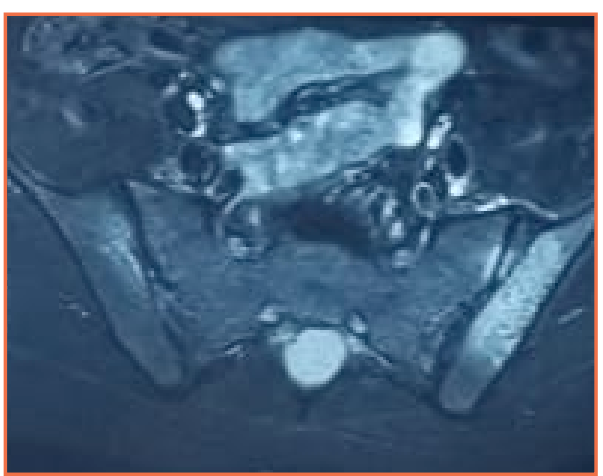

Figure 2. Asymmetric BMD in sacroiliacs joint.

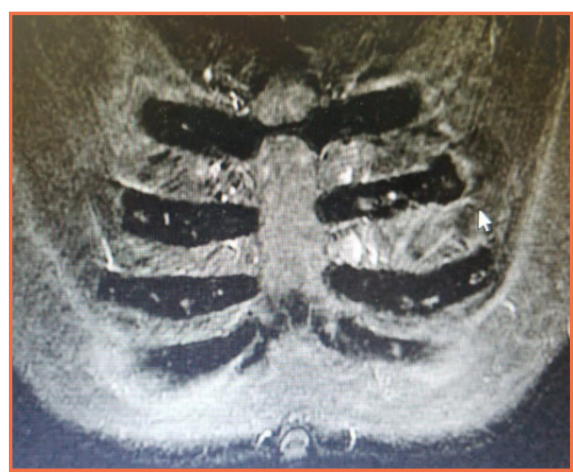

Figure 3. Involvement of the third and fourth sternocostal joint.

\section{CONCLUSION}

SAPHO patients can be a diagnostic challenge, especially regarding the differential diagnosis with spondyloarthritis; however, the fact that it is a rare disease, without the possibility of acceptable randomized studies makes it a condition that may not respond to the treatments described and, in consequence, could possibly bring major functional, labor and quality of life impairment. 